\title{
GENERIC SECTIONS OF ESSENTIALLY ISOLATED DETERMINANTAL SINGULARITIES
}

\author{
JEAN-PAUL BRASSELET, NANCY CHACHAPOYAS AND MARIA A. S. \\ RUAS
}

\begin{abstract}
We study the essentially isolated determinantal singularities (EIDS), defined by W. Ebeling and S. Gusein-Zade 8], as a generalization of isolated singularity. We prove in dimension 3 a minimality theorem for the Milnor number of a generic hyperplane section of an EIDS, generalizing previous results by J. Snoussi in dimension 2. We define strongly generic hyperplane sections of an EIDS and show that they are still EIDS. Using strongly general hyperplanes, we extend a result of Lê D. T. concerning constancy of the Milnor number.
\end{abstract}

\section{INTRODUCTION}

In this work, we study the essentially isolated determinantal singularities (EIDS), which have been defined by W. Ebeling and S. M. Gusern-Zade in 8. This type of singularities is a natural generalization of isolated ones. A generic determinantal variety $M_{m, n}^{t}$ is the subset of the space of $m \times n$ matrices, given by the matrices of rank less than $t$, where $t \leqslant \min \{m, n\}$. A variety $X \subset \mathbb{C}^{N}$ is determinantal if $X$ is the pre-image of $M_{m, n}^{t}$ by a holomorphic function $F: \mathbb{C}^{N} \rightarrow M_{m, n}$ with the condition that the codimension of $X$ in $\mathbb{C}^{N}$ is the same as the codimension of $M_{m, n}^{t}$ in $M_{m, n}$.

Determinantal varieties have isolated singularities if $N \leqslant(m-t+$ $2)(n-t+2)$ and they admit smoothing if $N<(m-t+2)(n-t+2)$. Several recent works investigate determinantal varieties with isolated singularities. The Milnor number of a determinantal surface was defined in [2, 13, 15] while the vanishing Euler characteristic of a determinantal variety was defined in [2, 13]. Other recent results on isolated determinantal varieties related to this paper appear in particular in [3, 6].

The first author is partially supported by CNPq grant no. 400580/2012-8. The second and third authors are partially supported by FAPESP grants no. 2010/09736-1, 2011/20082-6 and 2014/00304-2. The third author is partially supported by CNPq grant no. 301474/2005-2. 
Jean-Paul Brasselet, Nancy Chachapoyas and Maria A. S. Ruas

In this work we study the set of limits of tangent hyperplanes to determinantal varieties. For determinantal surfaces in $\mathbb{C}^{4}$ and 3-fold singularities in $\mathbb{C}^{5}$, we characterize these hyperplanes by the fact that the Milnor number of their sections with the surface in the first case or the 3-dimensional determinantal variety in the second case, is not minimum. The first case follows as a consequence of results of J. Snoussi in [16].

We also prove that given a $d$-dimensional EIDS $X$, given $H$ and $H^{\prime}$ strongly general hyperplanes to $X$ (Definition 4.2), there are $d-2$ linear planes $P \subset H$ and $P^{\prime} \subset H^{\prime}$ contained in $H$ and $H^{\prime}$, such that the Milnor number of the surfaces $X \cap P$ and $X \cap P^{\prime}$ are equal. In the case that the generic section is a curve the result has been already proved by Lê D. T. in [10].

\section{ESSEntially isolated Determinantal SingUlarity}

In this section we give the definition and basic results on essentially isolated determinant singularities, following [8].

We denote by $M_{m, n}$ the set of matrices $m \times n$ with complex entries.

Definition 2.1. For all $t, 1 \leqslant t \leqslant \min \{m, n\}$, let $M_{m, n}^{t}$ be the subset of $M_{m, n}$ whose elements are matrices of rank less than $t$ :

$$
M_{m, n}^{t}=\left\{A \in M_{m, n} \mid \operatorname{rank}(A)<t\right\} .
$$

This set is a singular variety of codimension $(m-t+1)(n-t+1)$ in $M_{m, n}$, called generic determinantal variety.

The singular set of $M_{m, n}^{t}$ is $M_{m, n}^{t-1}$. The partition of $M_{m, n}^{t}$ defined by

$$
M_{m, n}^{t}=\cup_{i=1, \ldots, t}\left(M_{m, n}^{i} \backslash M_{m, n}^{i-1}\right)
$$

is a Whitney stratification [1].

Let $F: \mathbb{C}^{N} \rightarrow M_{m, n}$ be a map defined by $F(x)=\left(f_{i j}(x)\right)$, whose entries are complex analytic functions defined on an open domain $U \subset$ $\mathbb{C}^{N}$.

Definition 2.2. The analytic variety $X=F^{-1}\left(M_{m, n}^{t}\right)$ is called determinantal variety of type $(m, n, t)$, if $\operatorname{codim} X=\operatorname{codim} M_{m, n}^{t}=$ $(m-t+1)(n-t+1)$.

A generic map $F$ intersects transversally the strata $M_{m, n}^{i} \backslash M_{m, n}^{i-1}$ of the variety $M_{m, n}^{t}$.

Definition 2.3. A point $x \in X=F^{-1}\left(M_{m, n}^{t}\right)$ is called essentially nonsingular if at this point the map $F$ is transversal to the corresponding stratum of the variety $M_{m, n}^{t}$ (that is, to $\left\{M_{m, n}^{i} \backslash M_{m, n}^{i-1}\right\}$, where $i=\operatorname{rank} F(x)+1)$. 
Generic sections of essentially isolated determinantal singularities

Definition 2.4. A germ $(X, 0) \subset\left(\mathbb{C}^{N}, 0\right)$ of a determinantal variety of type $(m, n, t)$ has an essentially isolated singular point at the origin (or is an essentially isolated determinantal singularity, EIDS) if it has only essentially nonsingular points in a punctured neighborhood of the origin in $X$.

An EIDS $X \subset \mathbb{C}^{N}$ has isolated singularity if and only if $N \leqslant(m-t+$ $2)(n-t+2)$. An EIDS with isolated singularity will be called isolated determinantal singularity, denoted by IDS.

We want to consider deformations of an EIDS that are themselves determinantal varieties of the same type.

Definition 2.5. An essential smoothing $\widetilde{X}_{s}$ of the $\operatorname{EIDS}(X, 0)$ is a subvariety lying in a neighborhood $U$ of the origin in $\mathbb{C}^{N}$ and defined by $\widetilde{X}_{s}=\widetilde{F}_{s}^{-1}\left(M_{m, n}^{t}\right)$ where $\widetilde{F}: U \times \mathbb{C} \rightarrow M_{m, n}$ is a perturbation of the germ $F$, with $\widetilde{F}_{s}(x)=\widetilde{F}(x, s), \widetilde{F}_{0}(x)=F(x)$ and such that $\widetilde{F}_{s}: U \rightarrow M_{m, n}$ is transversal to all strata $M_{m, n}^{i} \backslash M_{m, n}^{i-1}$.

An essential smoothing is in general not smooth (when $N \geq(m-$ $t+2)(n-t+2))$ as we see in the following theorem.

Theorem 2.1. [18] Let $(X, 0) \subset\left(\mathbb{C}^{N}, 0\right)$ be the germ of a determinantal variety with isolated singularity at the origin. Then, $X$ has a smoothing if and only if $N<(m-t+2)(n-t+2)$.

The singular set of the essential smoothing $\widetilde{X}_{s}$ is $\widetilde{F}_{s}^{-1}\left(M_{m, n}^{t-1}\right)$. Since $\widetilde{F}$ is transversal to the strata of the Whitney stratification $M_{m, n}^{t}$, the partition $\widetilde{X}_{s}=\cup_{1 \leq i \leq t} \widetilde{F}_{s}^{-1}\left(M_{m, n}^{i} \backslash M_{m, n}^{i-1}\right)$ is a Whitney stratification of $\widetilde{X}_{s}$.

Example 1. Let $X=F^{-1}\left(M_{2,3}^{2}\right)$, where

$$
\begin{aligned}
F: \mathbb{C}^{4} & \rightarrow M_{2,3} \\
(x, y, z, w) & \mapsto\left(\begin{array}{ccc}
z & y+w & x \\
w & x & y
\end{array}\right)
\end{aligned}
$$

The following matrix defines an essential smoothing $\widetilde{X}_{s}=\widetilde{F}_{s}^{-1}\left(M_{2,3}^{2}\right)$ of $X$

$$
\begin{aligned}
\widetilde{F}: \quad \mathbb{C}^{4} \times \mathbb{C} & \rightarrow M_{2,3} \\
(x, y, z, w, s) & \mapsto\left(\begin{array}{ccc}
z & y+w & x+s \\
w & x & y
\end{array}\right)
\end{aligned}
$$

In this case the essential smoothing $\tilde{X}_{s}$ is a smoothing. 
Jean-Paul Brasselet, Nancy Chachapoyas and Maria A. S. Ruas

\section{LÊ-GREUEL FORMUla TYPE FOR IDS With SMOOTHING}

We review in this section some results about isolated singularities admitting smoothing following [15].

Let $(X, 0) \subset\left(\mathbb{C}^{N}, 0\right)$ be the germ of a $d$-dimensional variety with isolated singularity at the origin. Suppose that $X$ has a smoothing. Then, there exists a flat family $\pi: \widetilde{X} \subset U \times \mathbb{C} \rightarrow \mathbb{C}$ such that the fiber $X_{s}=\pi^{-1}(s)$ is smooth for all $s \neq 0$ and $X_{0}=X$.

Let $p:(X, 0) \rightarrow \mathbb{C}$ be a complex analytic function defined in $X$ with isolated singularity at the origin. Let us consider a function

$$
\begin{aligned}
\tilde{p}: \mathbb{C}^{N} \times \mathbb{C} & \rightarrow \mathbb{C} \\
(x, s) & \mapsto \widetilde{p}(x, s),
\end{aligned}
$$

such that $\tilde{p}(x, 0)=p(x)$ and for all $s \neq 0, \widetilde{p}(\cdot, s)=p_{s}$ is a Morse function on $X_{s}$.

Thus we have the following diagram

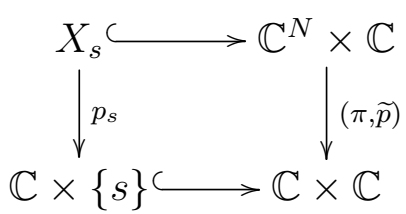

Proposition 3.1. [15, Proposition 4.1] Let $X$ be a d-dimensional variety with isolated singularity at the origin admitting smoothing and $p_{s}: X_{s} \rightarrow \mathbb{C}, p_{s}=\widetilde{p}(\cdot, s)$ as above. Then,

(a) If $s \neq 0, X_{s} \simeq p_{s}-1(0) \dot{\cup}\{$ cells of dimension $d\}$,

(b) $\chi\left(X_{s}\right)=\chi\left(p_{s}^{-1}(0)\right)+(-1)^{d} n_{0}$,

where $n_{0}$ is the number of critical points of $p_{s}$ and $\chi\left(X_{s}\right)$ denotes the Euler characteristic of $X_{s}$.

The invariant $n_{0}$ is related to the polar multiplicity of $X, m_{d}(X)$ ([15], see also [4]), in the following way:

Definition 3.1. (The $d$-Polar multiplicity) Let $X, \widetilde{X}, p$ and $\widetilde{p}$ as above.

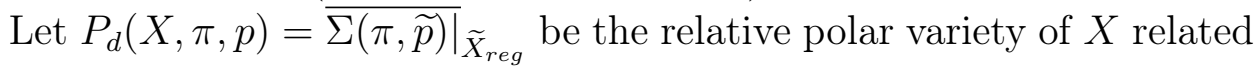
to $\pi$ and $p$. We define $m_{d}(X, \pi, p)=m_{0}\left(P_{d}(X, \pi, p)\right)$.

In general $m_{d}(X, \pi, p)$ depends on the choices of $\widetilde{X}$ and $\tilde{p}$. When the variety $X$ has a unique smoothing $\widetilde{X}$, then $m_{d}(X, \pi, p)$ depends only on $X$ and $p$. If $p$ is a generic linear embedding, $m_{d}(X, p)$ is an invariant of the EIDS $X$, denoted by $m_{d}(X)$.

Proposition 3.2. [15] Under the conditions of Proposition 3.1, $n_{0}=$ $m_{d}(X)$. 
Generic sections of essentially isolated determinantal singularities

Theorem 3.3. [7] Let $X_{s}$ be a smoothing of a normal isolated singularity, then $b_{1}\left(X_{s}\right)=0$.

Let $X$ be a determinantal variety of type $(m, n, t)$ in $\mathbb{C}^{N}$ with $N<$ $(m-t+2)(n-t+2)$. Then $X$ has isolated singularity and admits smoothing.

Definition 3.2. [15] Let $X$ be a determinantal surface in $\mathbb{C}^{N}$, with isolated singularity at the origin. The Milnor number of $X$, denoted by $\mu(X)$, is defined as the second Betti number of the generic fiber $X_{s}$,

$$
\mu(X)=b_{2}\left(X_{s}\right) .
$$

The following result appears in [2, 13, 15], for determinantal surfaces $X \subset \mathbb{C}^{4}$, but it also holds for any determinantal surface with isolated singularity in $\mathbb{C}^{N}$ admitting smoothing.

Proposition 3.4. Let $(X, 0) \subset\left(\mathbb{C}^{N}, 0\right)$ be the germ of a determinantal surface in $\mathbb{C}^{N}$ with isolated singularity at the origin admitting smoothing. Let $p:\left(\mathbb{C}^{N}, 0\right) \rightarrow(\mathbb{C}, 0)$ be a linear function whose restriction to $X$ has an isolated singularity at the origin. Then one has the Lê-Greuel formula

$$
\mu(X)+\mu\left(X \cap p^{-1}(0)\right)=m_{2}(X) .
$$

When $d=\operatorname{dim} X>2$, the Betti numbers $b_{i}(X), 2 \leqslant i<d$ are not necessarily zero (see [3]). In [2, 13, the authors define the vanishing Euler characteristic of varieties admitting smoothing.

Definition 3.3. [13] Let $(X, 0) \subset\left(\mathbb{C}^{N}, 0\right)$ be an IDS such that $N<$ $(m-t+2)(n-t+2)$. The vanishing Euler characteristic is defined by

$$
\nu(X)=(-1)^{d}\left(\chi\left(X_{s}\right)-1\right),
$$

where $X_{s}$ is a smoothing of $X$ and $\chi\left(X_{s}\right)$ is the Euler characteristic of $X_{s}$.

Theorem 3.5. [13] Let $(X, 0) \subset\left(\mathbb{C}^{N}, 0\right)$ be an IDS such that $N<$ $(m-t+2)(n-t+2)$ and let $p:\left(\mathbb{C}^{N}, 0\right) \rightarrow(\mathbb{C}, 0)$ be a linear projection whose restriction to $X$ has isolated singularity at the origin. Then,

$$
\nu(X)+\nu\left(X \cap p^{-1}(0)\right)=m_{d}(X)
$$

Remark. When $d=2$, then $\nu(X)=\mu(X)$.

Example 2. [14] Let $X=F^{-1}\left(M_{2,3}^{2}\right) \subset \mathbb{C}^{4}$ be the variety defined by:

$$
\begin{aligned}
& F: \quad \mathbb{C}^{4} \quad \rightarrow \quad M_{2,3} \\
& \left.(x, y, z, w) \mapsto \begin{array}{ccc}
z & y+w & x \\
w & x & y
\end{array}\right) .
\end{aligned}
$$


Jean-Paul Brasselet, Nancy Chachapoyas and Maria A. S. Ruas

The following matrix defines an essential smoothing $\widetilde{X}_{s}=\widetilde{F}_{s}^{-1}\left(M_{2,3}^{2}\right)$ of $X$

$$
\begin{aligned}
\widetilde{F}: \quad \mathbb{C}^{4} \times \mathbb{C} & \rightarrow M_{2,3} \\
(x, y, z, w, s) & \mapsto\left(\begin{array}{ccc}
z & y+w & x+s \\
w & x & y
\end{array}\right)
\end{aligned}
$$

Consider $p: \mathbb{C}^{4} \rightarrow \mathbb{C}$ given by $p(x, y, z, w)=w$, then it follows that $m_{2}(X)=3$ and $\mu\left(X \cap p^{-1}(0)\right)=2$, then $\mu(X)=1$.

\section{General and strongly General hyperplanes}

The main goal of this section is to define general and strongly general hyperplanes over an EIDS in order to extend previous results by J. Snoussi and Lê D. T. in the following sections. The two definitions are equivalent when the variety has an isolated singularity. The sections defined by the intersection of the IDS (respectively EIDS) and the general hyperplane (respectively strongly general hyperplane) determine another IDS (respectively EIDS).

Definition 4.1. The hyperplane $H \subset \mathbb{C}^{N}$, given by the kernel of the linear function $p: \mathbb{C}^{N} \rightarrow \mathbb{C}$ is called general to $X$ at 0 if $H$ is transversal to all limits $T$ of tangent spaces to the regular part of $X$.

Proposition 4.1. [16] A hyperplane $H$ is limit of tangent hyperplanes to $X$ at 0 , if only if $H$ is not a general hyperplane.

Example 3. Let $X$ be the swallowtail surface, given in Figure 1. $X$ is the surface in $\mathbb{C}^{3}$ defined by the zeros of

$$
256 z^{3}-27 x^{4}-128 z^{2} y^{2}+144 z x^{2} y+16 z y^{4}-4 x^{2} y^{3}=0 .
$$

The set of the limits of tangent hyperplanes to $X$ at zero is given by the hyperplane $z=0$ (see for example [12]).

Any hyperplane different of the hyperplane $z=0$ is general, in particular the plane $H=\{(x, y, z): x=0\}$ is general, but $H$ is not transversal to the limits of lines tangent to the strata of dimension 1.

This example motivates the following definition:

Definition 4.2. Let $X \subset \mathbb{C}^{N}$ be a d-dimensional analytic complex variety, and let $\left\{V_{\lambda}\right\}_{\lambda \in \Lambda}$ be a stratification of $X$. The hyperplane $H \subset$ $\mathbb{C}^{N}$ is called strongly general at the origin if it is general and there exists a neighborhood $U$ of 0 such that for all strata $V_{\lambda}$ of $X$, with $0 \in \bar{V}_{\lambda}$, we have that $H$ is transverse $V_{\lambda}$ at $x$, for all $x \in U \backslash\{0\}$.

Theorem 4.2. Let $(X, 0) \subset\left(\mathbb{C}^{N}, 0\right)$ be an EIDS of type $(n, m, t)$. If $H \subset \mathbb{C}^{N}$ is a strongly general hyperplane at the origin, then $X \cap H$ is $a(d-1)$-dimensional EIDS in $\mathbb{C}^{N-1}$ of the same type. 
Generic sections of essentially isolated determinantal singularities

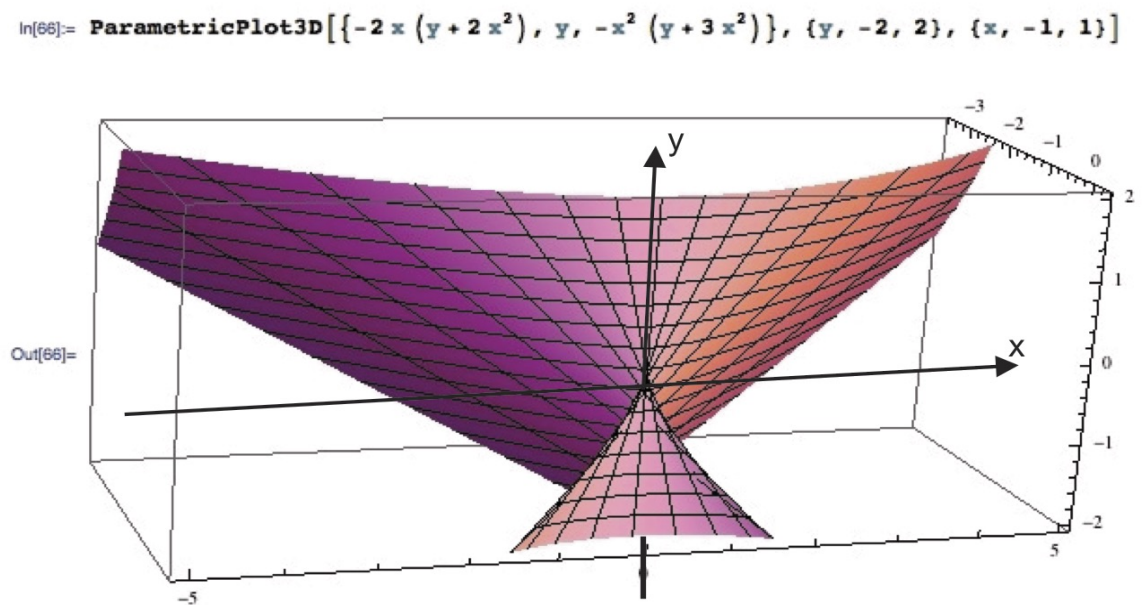

FiguRE 1. Swallowtail surface

Proof: Let $F: \mathbb{C}^{N} \rightarrow M_{m, n}$ be a function defining $X=F^{-1}\left(M_{m, n}^{t}\right)$. As $X$ is an EIDS, then $F$ is transversal to the strata of $M_{m, n}^{t}$ in $U \backslash\{0\}$, where $U$ is a sufficiently small neighborhood of the origin. By hypothesis $H$ is transversal to the strata of $X$ outside the origin. Let $i: \mathbb{C}^{N-1} \rightarrow \mathbb{C}^{N}$ be a linear embedding such $i\left(\mathbb{C}^{N-1}\right)=H$. Then $F \circ i: \mathbb{C}^{N-1} \rightarrow M_{m, n}$ is transversal to all the strata of $M_{m, n}^{t}$ outside the origin, so $X \cap H=(F \circ i)^{-1}\left(M_{m, n}^{t}\right) \subset \mathbb{C}^{N-1}$ is an EIDS of the same type of $X$ and $\operatorname{dim}(X \cap H)=d-1$ in $\mathbb{C}^{N-1}$.

\section{Minimality of the Milnor nUmber}

The minimality of the Milnor number of generic sections of hypersurfaces with isolated singularities was studied by B. Teissier [17] and J.-P. Henry and Lê D. T. [9]. T. Gaffney [5] proved the result for ICIS and J. Snoussi considered the case of normal surfaces in $\mathbb{C}^{N}$.

In the following we denote by $|Z|$ the reduction of the variety $Z$ and by $C_{X, x}$ the tangent cone to $X$ at $x$ (see [16]).

Proposition 5.1. [16, Theorem 4.2] Let $X \subset \mathbb{C}^{N}$ be the germ of a normal analytic surface. A hyperplane $H$ in $\mathbb{C}^{N}$ that does not contain any irreductible component of the tangent cone $\left|C_{X, 0}\right|$ is general to $X$ at 0 if and only if the section $X \cap H$ is reduced and the Milnor number $\mu(X \cap H)$ is minimum.

Proposition 5.2. [10] Let $X \subset \mathbb{C}^{N}$ be a complex analytic variety of dimension $d$ and let $L$ be an affine subspace of $\mathbb{C}^{N}$ of codimension equal 
Jean-Paul Brasselet, Nancy Chachapoyas and Maria A. S. Ruas

to the dimension of $C_{X, x}$ in $\mathbb{C}^{N}$ such that $L \cap C_{X, x}=\{x\}$. Consider the projection $\pi: \mathbb{C}^{N} \rightarrow \mathbb{C}^{d}$ such that $\mathrm{ker} \pi=L$. Consider $\Delta_{L}$ the discriminant of $\pi$ restricted to $X$. Let $H_{0}$ be a hyperplane of $\mathbb{C}^{d}$ which is not a limit of tangent hyperplanes to the discriminant $\Delta_{L}$. Then, the hyperplane $H$ given by the inverse image $H=\pi^{-1}\left(H_{0}\right)$ is not limit of tangent hyperplanes to $X$.

Proposition 5.3. [11, Corollary 2.3.2.1] Let $X \subset \mathbb{C}^{N}$ be a reduced analytic variety, $c$ an integer and $E$ be a vector space in $\mathbb{C}^{N}$ of codimension $c$ given by the intersection of $c$ hyperplanes $H_{1}, H_{2}, \ldots, H_{c}$ such that each $H_{i}$ is not tangent to $\left|X \cap H_{1} \cap H_{2} \cap \cdots \cap H_{i-1}\right|$, for $1 \leqslant i \leqslant c$. Then the multiplicity at $x$ of the polar variety $P_{k}(|X \cap E|)$ is equal to the multiplicity at $x$ of the polar varieties $P_{k}(X)$ for $0 \leqslant k \leqslant d-c-1$.

We extend Proposition 5.1 to 3-determinantal varieties with isolated singularities.

Theorem 5.4. Let $(X, 0) \subset\left(\mathbb{C}^{N}, 0\right)$ be the germ of a 3-dimensional determinantal variety with isolated singularity and $H$ a hyperplane in $\mathbb{C}^{N}$. Suppose that $X \cap H$ has an isolated singular point at the origin, then the following conditions are equivalent.

(i) $H$ is general to $X$ at 0 .

(ii) $\mu(X \cap H)$ is minimum and $\mu\left(X \cap H \cap H^{\prime}\right)$ is minimum for all $H^{\prime}$ general to $X$ and to $X \cap H$.

Proof: Suppose that $p: \mathbb{C}^{N} \rightarrow \mathbb{C}$ is general to $X$ at the origin, $H=p^{-1}(0)$. Then $X \cap H$ is a determinantal surface with isolated singularity at the origin. Then there exists a Zariski open set $\Omega \subset \mathbb{P}^{N-1}$ such that $\forall H^{\prime} \in \Omega$, with $H^{\prime}=\operatorname{ker} p^{\prime}, p^{\prime}: \mathbb{C}^{N} \rightarrow \mathbb{C}$ a linear map, we have that:

(1) $p$ is general to $X$ at the origin and $p$ is general to $X \cap H^{\prime}$ at the origin.

(2) $\mu\left(X \cap H^{\prime}\right)$ is minimum.

Applying Lê- Greuel type formula (1) of Proposition 3.4 for the surfaces $X \cap H$ and $X \cap H^{\prime}$, we have:

$$
\begin{gathered}
\mu(X \cap H)+\mu\left(X \cap H \cap H^{\prime}\right)=m_{2}(X \cap H) \\
\mu\left(X \cap H^{\prime}\right)+\mu\left(X \cap H \cap H^{\prime}\right)=m_{2}\left(X \cap H^{\prime}\right)
\end{gathered}
$$

As $H$ and $H^{\prime}$ are general to $X$ at 0 , it follows by Proposition 5.3 that $m_{2}(X \cap H)=m_{2}\left(X \cap H^{\prime}\right)=m_{2}(X)$. Then, equations (3) and (4) imply that $\mu(X \cap H)=\mu\left(X \cap H^{\prime}\right)$, that is $\mu(X \cap H)$ is minimum. 


\section{Generic sections of essentially isolated determinantal singularities}

Let $H^{\prime}$ and $H^{\prime \prime}$ be general hyperplanes to $X$ at the origin, defined by the kernels of $p^{\prime}$ and $p^{\prime \prime}$, such that $\mu\left(X \cap H^{\prime} \cap H^{\prime \prime}\right)$ is minimum. Then, the following Lê- Greuel type formulas holds for $X \cap H$ and $X \cap H^{\prime}$.

$$
\begin{aligned}
\mu(X \cap H)+\mu\left(X \cap H \cap H^{\prime}\right) & =m_{2}(X \cap H)=m_{2}(X) \\
\mu\left(X \cap H^{\prime}\right)+\mu\left(X \cap H^{\prime} \cap H^{\prime \prime}\right) & =m_{2}\left(X \cap H^{\prime}\right)=m_{2}(X)
\end{aligned}
$$

As $\mu(X \cap H)=\mu\left(X \cap H^{\prime}\right)$ it follows that $\mu\left(X \cap H \cap H^{\prime}\right)=\mu(X \cap$ $\left.H^{\prime} \cap H^{\prime \prime}\right)$.

Conversely, let $\pi=\left(p, p^{\prime}, p^{\prime \prime}\right)$ be the map $\mathbb{C}^{N} \rightarrow \mathbb{C}^{3}$, with $H=\operatorname{ker} p$, $H^{\prime}=\operatorname{ker} p^{\prime}$ and $H^{\prime \prime}=\operatorname{ker} p^{\prime \prime}$, such that $\operatorname{ker} \pi \cap C_{X, 0}=\{0\}$ and $m_{0}(X)=$ $\left.\operatorname{deg} \pi\right|_{X}$. Consider $\pi^{\prime}: \mathbb{C}^{3} \rightarrow \mathbb{C}^{2}$ such that $\left.\pi^{\prime} \circ \pi\right|_{X \cap H}=\left(p^{\prime}, p^{\prime \prime}\right)$, $\operatorname{ker}\left(\pi^{\prime} \circ \pi\right) \cap C_{X \cap H}=\{0\}$ and $m_{0}(X)=m_{0}(X \cap H)=\left.\operatorname{deg} \pi^{\prime} \circ \pi\right|_{X \cap H}$. Then

$$
\begin{gathered}
\mu\left(X \cap H \cap H^{\prime}\right)+\operatorname{deg} \pi-1=m_{1}\left(X \cap H \cap H^{\prime}\right) \\
\mu\left(X \cap H^{\prime} \cap H^{\prime \prime}\right)+\operatorname{deg} \pi-1=m_{1}\left(X \cap H^{\prime} \cap H^{\prime \prime}\right) .
\end{gathered}
$$

We have that $m_{1}(X)=m_{1}\left(X \cap H \cap H^{\prime}\right)$. On the other hand, it follows from [16, Lema 4.3] that

$$
\mu\left(X \cap H \cap H^{\prime}\right)+\operatorname{deg} \pi-1=\left(\Delta_{\pi^{\prime} \circ \pi} \cdot\left(\pi^{\prime} \circ \pi\right)\left(X \cap H \cap H^{\prime}\right)\right)_{0},
$$

where the notation $(a \cdot b)_{0}$ indicates the intersection multiplicity of $a$ and $b$. Then $m_{1}\left(X \cap H \cap H^{\prime}\right)=\left(\Delta_{\pi^{\prime} \circ \pi} \cdot\left(\pi^{\prime} \circ \pi\right)\left(X \cap H \cap H^{\prime}\right)\right)_{0}$ implies that $\left(\pi^{\prime} \circ \pi\right)\left(X \cap H \cap H^{\prime}\right)$ is not limit of tangent hyperplanes to $\Delta_{\pi^{\prime} \circ \pi}$. Then by Proposition 5.2, we have that $\pi(X \cap H)$ is not limit of tangent hyperplanes to $\Delta_{\pi}$. It follows from Proposition 5.2 that $H$ is not limit of tangent hyperplanes to $X$.

Example 4. Let $X \subset \mathbb{C}^{5}$ be a 3-determinantal variety with isolated singularity defined by

$$
\begin{aligned}
& F: \quad \mathbb{C}^{5} \quad \rightarrow \quad M_{2,3} \\
& (x, y, z, w, v) \mapsto\left(\begin{array}{ccc}
x & y & z \\
w & v & x^{2}+y^{2}
\end{array}\right)
\end{aligned}
$$

Let $H$ and $H^{\prime}$ be hyperplanes given by the kernels of $p(x, y, z, w, v)=$ $w-z$ and $p^{\prime}(x, y, z, w, v)=x-v$. The surfaces $X \cap H$ and $X \cap H^{\prime}$ are represented by the following matrices

$$
\left(\begin{array}{ccc}
x & y & z \\
z & v & x^{2}+y^{2}
\end{array}\right) \text { and }\left(\begin{array}{ccc}
x & y & z \\
w & x & y^{2}
\end{array}\right) \text { respectively. }
$$

It follows (see [15]) that $\mu(X \cap H)=4$ and $\mu\left(X \cap H^{\prime}\right)=2$, and that $\mu\left(X \cap H^{\prime}\right)=2$ is the minimum Milnor number. 
Jean-Paul Brasselet, Nancy Chachapoyas and Maria A. S. Ruas

\section{Sections of EIDS}

In this section, we show that when $X$ is an EIDS of dimension $d>2$ it is possible to associate to $X$ a determinantal surface $Y$ whose Milnor number is an invariant of $X$.

Proposition 6.1. [10] Let $H$ and $H^{\prime}$ be hyperplanes in $\mathbb{C}^{N}$ that are not limits of tangent hyperplanes to $(X, 0)$. Then there exist planes $Q$ and $Q^{\prime}$ in $H$ and $H^{\prime}$ respectively such that the reduced curves $|X \cap Q|$ and $\left|X \cap Q^{\prime}\right|$ have the same Milnor number.

The following result is a generalization of a result of Lê D. T.

Theorem 6.2. Let $X^{d} \subset \mathbb{C}^{N}$ be an EIDS and let $H, H^{\prime}$ be strongly general hyperplanes to $(X, 0)$ at the origin. Then $H$ and $H^{\prime}$ contain $P$ and $P^{\prime}$ respectively such that $\operatorname{codim} P=\operatorname{codim} P^{\prime}=d-2$, for which the determinantal surfaces $X \cap P$ and $X \cap P^{\prime}$ satisfy the following conditions:

a) $X \cap P$ and $X \cap P^{\prime}$ have isolated singularity.

b) $X \cap P$ and $X \cap P^{\prime}$ admit smoothing.

c) $\mu(X \cap P)=\mu\left(X \cap P^{\prime}\right)$.

\section{Proof:}

a) We can consider $P=H \cap \cdots \cap H_{d-3} \subset H$ and $P^{\prime}=H^{\prime} \cap \cdots \cap$ $H_{d-3}^{\prime} \subset H^{\prime}$ such that, for all $i, H_{i}$ and $H_{i}^{\prime}$ are strongly general for $X \cap H \cap H_{1} \cap \cdots \cap H_{i-1}$ and $X \cap H^{\prime} \cap H_{1}^{\prime} \cap \cdots \cap H_{i-1}^{\prime}$ respectively. By proposition 4.2, it follows that $X \cap P \subset \mathbb{C}^{N-d+2}$ and $X \cap P^{\prime} \subset$ $\mathbb{C}^{N-d+2}$ are EIDS. As codim $X<(m-t+2)(n-t+2)-2$ then $X \cap P$ and $X \cap P^{\prime}$ are determinantal surfaces with isolated singularity.

b) Let $\mathcal{X}$ be an essential smoothing of $X$, such that $H_{i} \times \mathbb{C}$ and $H_{i}^{\prime} \times \mathbb{C}$ are strongly general hyperplanes for $\mathcal{X} \cap(H \times \mathbb{C}) \cap\left(H_{1} \times\right.$ $\mathbb{C}) \cap \cdots \cap\left(H_{i-1} \times \mathbb{C}\right)$ and $\mathcal{X} \cap\left(H^{\prime} \times \mathbb{C}\right) \cap\left(H_{1}^{\prime} \times \mathbb{C}\right) \cap \ldots\left(\cap H_{i-1}^{\prime} \times \mathbb{C}\right)$ respectively. In fact, $\mathcal{X} \cap \widetilde{P}$ and $\mathcal{X} \cap \widetilde{P}^{\prime}$, with $\widetilde{P}=P \times \mathbb{C}$ and $\widetilde{P}^{\prime}=P^{\prime} \times \mathbb{C}$ are the smoothings of $X \cap P$ and $X \cap P^{\prime}$ respectively.

c) The hyperplanes $H$ and $H^{\prime}$ contain planes $Q=P \cap H^{\prime}$ and $Q^{\prime}=P^{\prime} \cap H$ given in Proposition 6.1. Then $X \cap Q$ and $X \cap Q^{\prime}$ are determinantal curves and by Proposition 6.1, we have that:

$$
\mu(X \cap Q)=\mu\left(X \cap Q^{\prime}\right) .
$$

Using Lê-Greuel type formula, Proposition 3.4, we get

$$
\begin{aligned}
\mu(X \cap P)+\mu(X \cap Q) & =m_{2}\left(X \cap P, p^{\prime}\right) \\
\mu\left(X \cap P^{\prime}\right)+\mu\left(X \cap Q^{\prime}\right) & =m_{2}\left(X \cap P^{\prime}, p\right) . \\
10 &
\end{aligned}
$$




\section{Generic sections of essentially isolated determinantal singularities}

We can apply Proposition 5.3 to the variety $\mathcal{X} \subset \mathbb{C}^{N} \times \mathbb{C}$, and to the hyperplanes $H_{i} \times \mathbb{C}$. By definition, $m_{2}\left(X \cap P, p^{\prime}\right)=$ $m_{2}\left(X \cap P^{\prime}, p\right)=m_{0}\left(P_{2}(\mathcal{X})\right)$, and $m_{2}\left(X \cap P^{\prime}, p\right)=m_{0}\left(P_{2}(\mathcal{X})\right)$.

Then $m_{2}\left(X \cap P, p^{\prime}\right)=m_{2}\left(X \cap P^{\prime}, p\right)=m_{0}\left(P_{2}(\mathcal{X})\right)$ and using (9) we have the result.

Example 5. Let $F: \mathbb{C}^{N} \rightarrow M_{2,3}$ be an analytic map, $N \geqslant 7$ defined by

$$
F(x, y)=\left(\begin{array}{ccc}
x_{1} & x_{2} & x_{3} \\
x_{4} & x_{5} & x_{6}+g(y)
\end{array}\right)
$$

where $x=\left(x_{1}, x_{2}, \ldots, x_{6}\right), y=\left(y_{1}, \ldots, y_{N-6}\right)$ and $g: \mathbb{C}^{N-6} \rightarrow \mathbb{C}$ is an analytic function with $g(0)=0$. Then $X=F^{-1}\left(M_{m, n}^{t}\right)$ is a CohenMacaulay codimension 2 singularity in $\mathbb{C}^{N}$. Let $P=\left\{(x, y) \mid x_{1}=\right.$ $\left.x_{5}, x_{2}=x_{6}, y=0\right\}$. Then $X \cap P$ is a determinantal surface in $\mathbb{C}^{4}$ defined by

$$
\left(\begin{array}{lll}
x_{1} & x_{2} & x_{3} \\
x_{4} & x_{1} & x_{2}
\end{array}\right)
$$

and $\mu(X \cap P)=1$ (see [14]).

\section{REFERENCES}

[1] E. Arbarello, M. Cornalba, P. A. Griffiths, and J. Harris. Geometry of algebraic curves. Vol. I, volume 267 of Grundlehren der Mathematischen Wissenschaften [Fundamental Principles of Mathematical Sciences]. Springer-Verlag, New York, 1985.

[2] J. Damon and B. Pike. Solvable groups, free divisors and nonisolated matrix singularities II: Vanishing topology. Geom. Topol., 18(2):911-962, 2014.

[3] A. Frühbis-Krüger and M. Zach. On the vanishing topology of isolated CohenMacaulay codimension 2 singularities. Preprint, 2015.

[4] T. Gaffney. Polar multiplicities and equisingularity of map germs. Topology, 32(1):185-223, 1993.

[5] T. Gaffney. Aureoles and integral closure of modules. In Stratifications, singularities and differential equations, II (Marseille, 1990; Honolulu, HI, 1990), volume 55 of Travaux en Cours, pages 55-62. Hermann, Paris, 1997.

[6] T. Gaffney and A. Rangachev. Pairs of modules and determinantal isolated singularities. Preprint, 2014.

[7] G.-M. Greuel and J. Steenbrink. On the topology of smoothable singularities. In Singularities, Part 1 (Arcata, Calif., 1981), volume 40 of Proc. Sympos. Pure Math., pages 535-545. Amer. Math. Soc., Providence, R.I., 1983.

[8] S. M. Guseľn-Zade and W. Ėbeling. On the indices of 1-forms on determinantal singularities. Tr. Mat. Inst. Steklova, 267(Osobennosti i Prilozheniya):119-131, 2009.

[9] J.-P. G. Henry and D. T. Lê. Limites d'espaces tangents. In Fonctions de plusieurs variables complexes, II (Sém. François Norguet, 1974-1975), pages 251-265. Lecture Notes in Math., Vol. 482. Springer, Berlin, 1975. 
Jean-Paul Brasselet, Nancy Chachapoyas and Maria A. S. Ruas

[10] D. T. Lê. Generic sections of singularities. In Singularity theory, pages 677-682. World Sci. Publ., Hackensack, NJ, 2007.

[11] D. T. Lê and B. Teissier. Limites d'espaces tangents en géométrie analytique. Comment. Math. Helv., 63(4):540-578, 1988.

[12] W. L. Marar, J. A. Montaldi, and M. A. S. Ruas. Multiplicities of zero-schemes in quasihomogeneous corank-1 singularities $\mathbf{C}^{n} \rightarrow \mathbf{C}^{n}$. In Singularity theory (Liverpool, 1996), volume 263 of London Math. Soc. Lecture Note Ser., pages 353-367. Cambridge Univ. Press, Cambridge, 1999.

[13] J. Nuño Ballesteros, B. Oréfice-Okamoto, and J. Tomazella. The vanishing Euler characteristic of an isolated determinantal singularity. Israel Journal of Mathematics, 197(1):475-495, 2013.

[14] M. S. Pereira. Variedades determinantais e singularidades de matrizes. $\mathrm{PhD}$ thesis, ICMC, 2010.

[15] M. S. Pereira and M. A. S. Ruas. Codimension two determinantal varieties with isolated singularities. Math. Scandinavica, 115, pages 161-172, 2014.

[16] J. Snoussi. Limites d'espaces tangents à une surface normale. Comment. Math. Helv., 76(1):61-88, 2001.

[17] B. Teissier. Cycles évanescents, sections planes, et conditions de Whitney. In Astérisque, volume 7-8, pages 285-362. 1973.

[18] J. Wahl. Smoothings of normal surface singularities. Topology, 20(3):219-246, 1981. 